\title{
Correntropy measures to detect daytime sleepiness from EEG signals
}

Umberto Melia $^{\mathrm{a} *}$, Marc Guaita ${ }^{\mathrm{b}, \mathrm{g}}$, Montserrat Vallverdúa ${ }^{\mathrm{a}}$, Josep M. Montserrat ${ }^{\mathrm{b}, \mathrm{ch}, \mathrm{l},}$, Isabel Vilaseca ${ }^{\mathrm{b}, \mathrm{d}, \mathrm{h}, \mathrm{l}}$, Manel

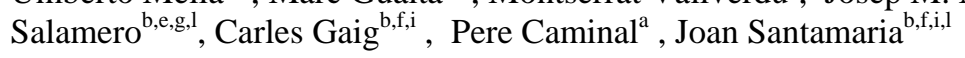

${ }^{a}$ Dept. ESAII, Centre for Biomedical Engineering Research, CIBER-BBN, Universitat Politècnica de Catalunya, Pau Gargallo 5, 08028, Barcelona, Spain

${ }^{\mathrm{b}}$ Multidisciplinary Sleep Disorders Unit, Hospital Clinic, Barcelona, Spain.

${ }^{\mathrm{c}}$ Department of Pneumology, Hospital Clinic, Barcelona, Spain.

${ }^{\mathrm{d}}$ Department of Otorhinolaryngology, Hospital Clinic, Barcelona, Spain.

${ }^{\mathrm{e}}$ Department of Psychiatry, Hospital Clinic, Barcelona, Spain.

${ }^{\mathrm{f}}$ Department of Neurology, Hospital Clinic, Barcelona, Spain.

'Institut d’ Investigació Biomèdica August Pi i Sunyer (IDIBAPS), Barcelona, Spain.

${ }^{\mathrm{h}}$ Ciber Enfermedades Respiratorias (CIBERES), Madrid, Spain.

${ }^{\mathrm{i} C i b e r ~ E n f e r m e d a d e s ~ N e u r o l o ́ g i c a s ~(C I B E R N E D), ~ B a r c e l o n a, ~ S p a i n . ~}$

${ }^{\mathrm{l}}$ Medical School, University of Barcelona

* Corresponding author: +34934017159 fax:+34934017045

e-mail: umberto.melia@upc.edu

\begin{abstract}
Excessive daytime sleepiness (EDS) is one of the main symptoms of several sleep related disorders with a great impact on the patient lives. While many studies have been carried out in order to assess daytime sleepiness, the automatic EDS detection still remains an open problem. In this work, a novel approach to this issue based on correntropy function analysis of EEG signals was proposed in order to detect patients suffering EDS. Multichannel EEG signals were recorded during five Maintenance of Wakefulness Tests (MWT) and Multiple Sleep Latency Tests (MSLT) alternated throughout the day from patients suffering from sleep disordered breathing (SDB). A group of 20 patients with EDS was compared with a group of 20 patients without daytime sleepiness (WDS), by analyzing 60-second EEG windows in waking state. Measures obtained from cross-correntropy function (CCORR) and auto-correntropy function (ACORR) were calculated in the EEG frequency bands: $\delta$, $0.1-4 \mathrm{~Hz} ; \theta, 4-8 \mathrm{~Hz} ; \alpha, 8-12 \mathrm{~Hz} ; \beta, 12-30 \mathrm{~Hz}$; total band TB, 0.1-45 Hz. These functions permitted to quantify the complexity signal properties and the non-linear couplings between different areas of the scalp. Statistical differences between EDS and WDS groups were mainly found in $\beta$ band during MSLT events ( $\mathrm{p}$-value<0.0001). WDS group presented more complexity than EDS in the occipital zone, while a stronger nonlinear coupling between occipital and frontal regions was detected in EDS patients than in WDS. At best, ACORR and CCORR measures yielded sensitivity and specificity above $80 \%$ and area under ROC curve (AUC) above 0.85 in classifying EDS and WDS patients. These performances represent an improvement respect classical EEG indices applied in the same database (sensitivity and specificity were never above $80 \%$ and AUC under 0.75 ).
\end{abstract}

Keywords - Biomedical signal processing, Complexity theory, Correntropy, Electroencephalography, Excessive daytime sleepiness. 


\section{Introduction}

Excessive daytime sleepiness (EDS) is a socially and clinically relevant problem (Ohayon 2008). EEG is often applied to measure sleepiness. Changes in the frequency and amplitude of the EEG correlate directly with behavioral performance measures, particularly on tasks which require sustained attention over long periods of time (Jung and Makeig 1994; Santamaria and Chiappa 1987; Makeig et al 1993; Andrews-Hanna et al 2010, Gusnard et al 2001, Vanhaudenhuyse et al 2010). In the clinical practice, the multiple sleep latency test (MSLT) (Carskadon, 1986) and the maintenance of wakefulness test (MWT) (Doghramji, 1997) are considered the gold standard to objectively evaluate EDS. They are based on visual analysis of changes in the frequency and amplitude of the EEG signal in order to detect EDS. These tests are relatively complex, require trained technicians to interpret the signal and are expensive to perform on daily routine. Because of that, simplified methods that easily evaluate objective EDS and that could be used broadly in clinical and real-life scenarios are needed

Several researchers have attempted to leverage these characteristics for the development of EEG based drowsiness algorithms using EEG feature extractions (Johnson et al 2011), EEG power spectral density (PSD) bandwidth comparisons (Lin et al 2008, Swarnkar et al 2008), increases latencies of event related potentials (Smith et al 2002), linear regression (Chiou et al 2006), stepwise linear discriminant functions, artificial neural networks (Subasi and Erçelebi 2005), and principal component analysis (Fu J et al 2008) and linear regression models (Lin et al 2005). In another work, Wilson and Bracewell (2000) developed a method for detecting the alert state by applying a wavelet-based preprocessing and an artificial neural network (ANN); Vuckovic et al. (2000) used ANN as an automatic classifier of alertness and drowsiness from EEG recordings on arbitrary normal subjects. More recently, Yeo et al (2009) applied support vector machines (SVM) as a classification tool to process EEG data for the detection of driving drowsiness. However, these algorithms present methodologies and results of mixed quality and weakness as small sample size, non-inclusion of sleep disorders, lack of cross validation analysis, task dependence/specificity, algorithm complexity, comparison with manual classification of EEG data that include consistency problems, and large number of channels required. Moreover, the study of sleep onset while driving differs from normal sleep studies as the passage from wakefulness to sleep is resisted and the subject struggles to maintain vigilance. Furthermore, since many features of EEG signals cannot be generated by linear models, it is generally argued that non-linear measures are likely to give more information than the ones obtained with conventional linear approaches.

In the last few years, nonlinear techniques have been used in neurophysiology to comprehend complex dynamics of the underlying processes (Jeong 2004, Sabeti et al 2009) or they have been used to detect nonlinear interactions (Alonso et al 2010). The fundamental assumption of nonlinear techniques is that EEG signal is generated by nonlinear deterministic processes with nonlinear coupling interactions between neuronal populations. Nonlinearity in the brain is introduced, even at the neuronal level (Andrzejak et al 2010), since the dynamical behavior of individual neurons is governed by threshold and saturation phenomena, large networks of interconnected neurons are likely candidates for self-organized criticality, which refers to large systems with local nonlinear interactions in which a slow build-up of some energy value is alternated with brief bursts of energy redistribution (Stam 2005).

In general, the most applied complexity measures found in the literature are attractor dimension (Pradhan and Sadasivan 1996), correlation dimension (Acharya et al 2005), Lyapunov exponent (Fell et al 1993), fractal dimension (Chouvarda et al 2011) and sample entropy (Chouvarda et al 2011). All complexity measures mentioned above depend on the setting of estimation parameters, namely embedding dimension, time delay of phase space reconstruction, prediction time horizon, and partitioning. All these parameters differ depending on the particular investigation aspects. These dimension based methods suppose a large signal size, besides that, the limited length of EEG time series available for sleepiness characterization disallows general dimension estimation methods.

Correntropy is a new concept introduced by the group of Principe (Santamaria et al 2006) to quantify similarity using the higher order statistics in random processes based on a reproducing kernel Hilbert space (RKHS) method (Santamaria et al 2006). By nonlinearly transforming the random processes into a high dimensional RKHS and computing the conventional correlation on the transformed signals, correntropy is sensitive to both the higher order statistical distribution information and temporal structure of the original random process. The ability to reflect nonlinear characteristics of the signal makes correntropy a wellqualified candidate for characterization of nonlinear dynamics. Another attractive property of the correntropy function is its robustness against impulsive noise. Besides that, the use of kernel methods makes the correntropy computationally efficient since it can be computed directly from the data.

This work introduces a novel approach to EDS problem based on nonlinear signal processing techniques. 
Correntropy functions were applied to multichannel EEG signals in order to detect patients with and without sleepiness before the sleep onset. The study was performed in patients suffering sleep disturbance undergoing the maintenance of wakefulness test (MWT) and multiple sleep latency test (MSLT). In the clinical practice, the MWT and MSLT are frequently used as sleep disorder diagnostic tools. They measure the time elapsed to fall asleep (sleep latency) in a soporiferous situation when the subject is instructed not to fall asleep (MWT) or with the instruction to try to fall asleep (MSLT). In other words, the MWT measures the resistance to fall asleep whereas the MSLT measures the capacity to fall asleep (Carskadon et al 1986, Richardson et al 1978, Thorpy et al 1992).

\section{Materials and Methodology}

\subsection{EEG Database}

The analyzed database belongs to the Multidisciplinary Sleep Disorders Unit of the Hospital Clínic (Barcelona, Spain). From a series of 98 consecutive patients with symptoms of SDB, 2 groups of 20 patients were selected consecutively based on mean sleep latencies from a MWT-MSLT research protocol: excessive daytime sleepiness (EDS) group (MWT < 20min and MSLT < 8min) and without daytime sleepiness (WDS) group (MWT $>20 \mathrm{~min}$ and MSLT $>8 \mathrm{~min}$ ). They were matched by age and gender. For each patient, 6 channels of EEG (F3,F4,C3,C4,O1,O2) referenced to linked earlobes (A1+A2)/2 were recorded at $256 \mathrm{~Hz}$ during MWT and MSLT. The MSLT and MWT consisted of a series of five nap opportunities during the day beginning approximately one hour after morning awakening, starting with the MWT and alternating each other throughout the day. The MSLT is performed with the subject lying down in bed in a comfortable position in a dark and quiet room with explicit instruction to try to fall asleep and the MWT with subjects semi-recumbent in a bed and with the instruction to stay awake. If no sleep occurred MWT and MSLT trials were ended after 40 and 20 minutes respectively, or after unequivocal sleep, defined as three consecutive epochs of stage 1 sleep, or one epoch of any other stage of sleep. Objective daytime sleepiness was measured from sleep latency defined as time from lights out to the first epoch of unequivocal sleep on each test (Carskadon et al 1986, Richardson et al 1978, Thorpy et al 1992).

The MWT and MSLT tests was scheduled similarly to previous works (Sangal et al 1992, Kingshot et al 1999), with the same order of tests for all patients and following the recommended setting conditions of the AASM (Littner et al 2005). In this way, we have evaluated 2 different aspects of daytime sleepiness throughout the same day: the ability to stay awake (MWT) and the facility to fall asleep (MSLT). The subjects in the EDS group were those that had both a low resistance to stay awake and a high tendency to fall asleep, whereas the WDS group had both a high resistance to fall asleep and a low tendency to fall asleep even in the appropriate circumstances.

Mean sleep latencies were chosen as the general measure of daytime sleepiness and for selecting the groups. This variable was calculated averaging the 5 individual MWT on the one hand and the 5 individual MSLT sleep latencies on the other, defining sleep latency as the time from lights out to the first epoch of unequivocal sleep. Patients from the EDS group (the sleepier group) had much shorter mean sleep latencies than the WDS group (the more alert group): MWT $(11.5 \pm 4.54 \mathrm{~min}$ versus $35.3 \pm 6.33 \mathrm{~min}$, p-value $<0.001)$ and MSLT $(4.4 \pm 1.96 \mathrm{~min}$ versus $11.66 \pm 2.41 \mathrm{~min}, \mathrm{p}$-value $<0.001)$. We can assume that mean values are reflecting the overall daytime sleepiness.

\subsection{EEG Preprocessing}

After the application of a FIR band pass filter of 50th order, with cut-off frequencies of $0.1-45 \mathrm{~Hz}$, the EEG channels were resampled at $128 \mathrm{~Hz}$. Then, the EEG was segmented using $60 \mathrm{~s}$ sliding windows with increments of 20 s calculated during the entire tests. The raw EEG was visually checked in order to avoid the analysis of EEG windows corrupted by strong artifacts. In general, the first $30 \mathrm{~s}$ of each MWT and MSLT trials were not taken into account because they were altered by movement artifacts due to patients trying to find their comfortable position. Furthermore, windows that contain high amplitude ocular artifacts were rejected and the subsequent windows were taken into account for the analysis. The selected windows were filtered into the characteristic frequency bands of the EEG signal: $\delta, 0.1-4 \mathrm{~Hz} ; \theta, 4-8 \mathrm{~Hz} ; \alpha, 8-12 \mathrm{~Hz} ; \beta, 12-30$ $\mathrm{Hz}$; total band $T B, 0.1-45 \mathrm{~Hz}$. 


\subsection{Correntropy Function}

Autocorrentropy function (ACORR) was used to describe the complexity of the EEG signals and crosscorrentropy function (CCORR) was applied between different EEG channels to assess brain connectivity. These techniques both detect linear and nonlinear statistical dependencies between time series, whereas the more standard correlation function measures only their linear dependence. Thus, indexes based on CCORR assess dynamical coupling or information transmission between two systems and when applied to the EEG they may be postulated to be measures of functional connectivity (Gunduz and Principe 2009, Xu et al 2007). The main goal of this work was to characterize two groups of patients with excessive daytime sleepiness (EDS) and without daytime sleepiness (WDS) by the analysis of EEG windows in waking state.

Correntropy is a similarity measure of signals, mapped nonlinearly into a feature space (Santamaria et al 2006). In essence, correntropy generalizes the autocorrelation function to nonlinear spaces. Let $\{(x(t), t \in T)$, be a stochastic process with $T$ being an index set. The auto-correntropy function is defined from $T \times T$ into $\mathrm{R}^{+}$ given by

$$
V_{x}(t, s)=E\left(\kappa\left(x_{t}-x_{s}\right)\right)=\int \kappa\left(x_{t}-x_{s}\right) p(x) d x
$$

$\kappa(\cdot)$ is a symmetric positive definite kernel function. For strictly stationary and ergodic random process:

$$
\widehat{V}(\tau)=\frac{1}{N} \sum_{n=1}^{N} \kappa(x(n)-x(n-\tau))
$$

Equation (2) is also called the auto-correntropy function (ACORR) due to the analogy with the autocorrelation of random processes and the property that its average over the lags is the Information Potential, i.e. the argument of Renyi's entropy (Xu et al 2007).

In the literature, sigmoidal, Gaussian, polynomial, and spline kernels are among the mostly used symmetric positive definite kernel functions in the area of machine learning, function approximation, density estimation, support vector machine, and others. A widely used kernel is the Gaussian kernel given by

$$
\kappa_{\sigma}\left(x, x^{\prime}\right)=\frac{1}{\sqrt{2 \pi \sigma}} e^{-\frac{(x-x)^{2}}{2 \sigma^{2}}}
$$

where $\sigma$ is the size of the kernel. The Gaussian kernel is symmetric and positive definite.

Therefore, the ACORR partially characterizes higher order statistics of random processes with a compact bivariate kernel function. The emphasis given to higher-order moments is controlled by the parameter $\sigma$, which in this study is determined by Silverman's rule (Xu et al 2007) of density estimation:

$$
\sigma=0.9 A N^{-1 / 5}
$$

In this rule, $N$ is the number of samples and $A$ is the smaller value between the standard deviation of the data samples and data interquartile range scaled by 1.34 . In the issue at hand, this technique provides a reasonable initial value since the signal is scalar. A powerful advantage of the ACORR is its robustness against impulsive noise. This advantage is due to the fact that when an outlier is present, the inner product in the feature space computed via the Gaussian kernel tends to be zero. In this way, ACORR is a symmetric positive function with a maximum at the origin $(1 / \sqrt{2 \pi \sigma})$.

In (Santamaria et al 2006) the Fourier transform of ACORR was introduced as the generalized power spectral density (PSD) and named correntropy spectral density (CSD). Its expression is given as follows:

$$
\operatorname{CSD}\left(e^{j \omega}\right)=\sum_{\tau=-\infty}^{\infty} V(\tau) e^{-j \omega \tau}
$$

Correntropy can also be defined for pairs of time series assuming the name of Cross-correntropy function (CCORR): 


$$
\widehat{V} x y(\tau)=\frac{1}{N} \sum_{n=1}^{N} \kappa(x(n)-y(n-\tau))
$$

CCORR is a nonlinear interdependence measure. Due to a positive definite kernel function, the CCORR implicitly maps the original random variables or time series into an infinite dimensional reproducing kernel Hilbert space which is uniquely induced by the centered CCORR function and essentially computes the cosine of the angle between the two transformed vectors (Santamaria et al 2006). CCORR is able to extract more information than the cross-correlation function in quantification of coupling and synchronization between interacting dynamical systems. Indexes defined on CCORR function of EEG signals can quantify the bilateral synchronization or couplings among the corresponding sensory areas of the brain (Xu et al 2007).

CCORR was calculated between pairs of EEG channels located over frontal, occipital and central regions (F3-C3, F4-C4, C3-O3, C4-O4), between central and occipital regions (O1-C3, O2-C4), across the central line (O1-F3, O2-F4) and between all pairs of inter-hemispheric channels. ACORR was calculated for the six EEG channels and normalized by the maximum value.

Several measures were calculated from ACORR and CCORR with respect to delay $\tau$ in order to quantify and extract the essential information contained on the functions: mean $(\mathrm{m})$, maximum $(\mathrm{max})$, minimum $(\mathrm{min})$, first relative maximum $(\max L)$, rate of decrease $(R D)$, first derivative $(F D)$ and the mean frequency of CSD $(C S D m F)$. The FD measure was calculated as the difference between the ACORR(0) and the ACORR(1), similarly FD measure for CCORR was calculated as the difference between the absolute maximum of the CCORR and the CCORR at subsequent $\tau$.

\subsection{Traditional EEG Measures}

In order to compare the correntropy measures with temporal and frequency linear measures, the following traditional measures were calculated in each channel:

- The standard deviation (std) of the EEG windows filtered in each band.

- The power spectral density (PSD) for each EEG window in TB band using the Welch method.

- The spectral power in each band $\left(P_{\delta}, P_{\theta}, P_{\alpha}, P_{\beta}\right)$ as the area under the PSD curve in each band normalized by the total PSD area.

- The mean frequency $(m F)$ in each band (TB, $\delta, \theta, \alpha, \beta$, ) as the centroid of the PSD curve.

- The spectral edge frequencies SEF50, SEF75 and SEF90 in each bands. The SEFX was calculated as the frequency below which $\mathrm{x} \%$ of the total EEG spectral power is located.

- The same measures applied to ACORR were calculated on autocorrelation function (Ac)

Furthermore, the following measures were calculated between all the combinations of the channels:

- The mean, the maximum value, the mF and SEF50, SEF75 and SEF90 of the coherence function $(C f)$ in each band.

- The same measures applied to CCORR were calculated on cross-correlation function (Cc)

\subsection{Analysis of the EEG Measures}

To pursue our goal, different studies were performed comparing the proposed measures calculated from EDS patients and from WDS patients.

Firstly, individual analysis of MSLT and MWT naps (study 1) was performed with measure calculated from the first $60 \mathrm{~s}$ window at the beginning of each MWT (MWT1, MWT2, MWT3, MWT4, MWT5) and MSLT (MSLT1, MSLT2, MSLT3, MSLT4, MSLT5).

Mean value of each measure was calculated for all MWT and all MSLT naps for each patient (study 2) in order to find the measures that can best characterize the two groups.

Then, in order to assess if the previous results were improved by the changes that two different situations (MWT and MSLT) produced in a single measure in the two groups (EDS and WDS), multi-variable discriminant functions (study 3) were built by combining a measure of study 1 calculated in MWT with the equivalent measure calculated in MSLT.

A period comprised between the $300 \mathrm{~s}$ before and 100s after sleep onset were used to focus on the moment of change that is common in both groups. Patients that did not fall asleep in a given nap were excluded from the analysis (study 4), in order to reduce the influence on the results of awake patients. 


\subsection{Statistical Analysis}

A non-parametric test, Mann-Whitney U-test, was applied and a significance level p-value $<0.05$ was taken into account. Measures that satisfy this condition were considered for building a discriminant function. The leaving-one-out method was performed as validation method. Sensitivity (Sen) and specificity (Spe) were calculated for testing the performance of the measures. The proportion of EDS patients correctly classified was counted by Sen and the proportion of WDS patients correctly classified by Spe.

Also the area under ROC curve $(A U C)$ was used to test the performance of the measures. The ROC curve was computed for the results of the predictions calculated with logistic regression classification using a generalized linear model. The model was built by fitting a generalized linear regression of the predicted classes on the measures, using normal distribution (McCullagh and Nelder 1990).

\section{Results}

To show the profiles of ACORR and CCORR we depict the best discriminatory measures obtained in this study. ACORR $(\tau)$ applied to O2 EEG channel in $\beta$ band exhibited transient oscillation and then decreased to nonzero stable values after a certain time delay (figure 1). It is not possible to visually denote different complexity behavior between the two EDS and WDS groups in the time domain but in the frequency domain (ACORR power spectrum $C S D(f)$ in figure $1 \mathrm{~b}$ ) it can be noted that EDS group presents higher power in low frequencies and lower power in high frequencies than WDS group.

In figure 2, profile of $\operatorname{CCORR}(\tau)$ calculated between F3-O2 EEG channel in $\beta$ band and its $\operatorname{CSD}(f)$ also exhibited transient oscillation and then decreased gradually round nonzero stable values after a certain time delay. It is possible to deduce that EDS group presents more nonlinear coupling than WDS group between F3$\mathrm{O} 2$ channels in $\beta$ band at different time scale in both MWT and MSLT tests. The power spectrum of CCORR presents a peak in the range of $\beta$ band that assumes the highest value in MSLT.

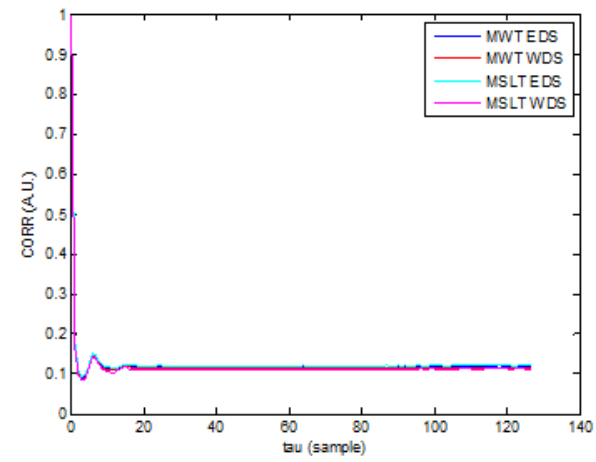

(a)

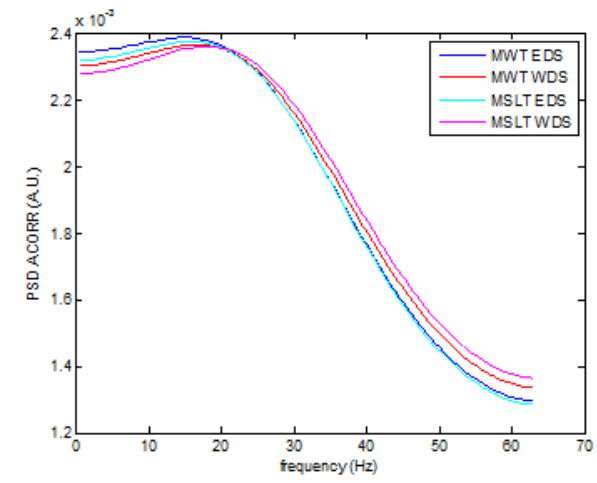

(b)

Fig. 1. Channel O2, $\beta$ band. Averaging of (a) $\operatorname{ACORR}(\tau)$ and (b) its $\operatorname{CSD}(f)$ with respect to all MWT and MSLT of all EDS and WDS patients.

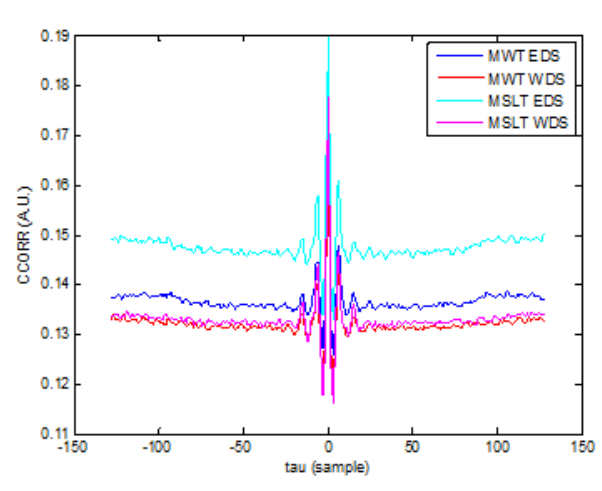

(a)

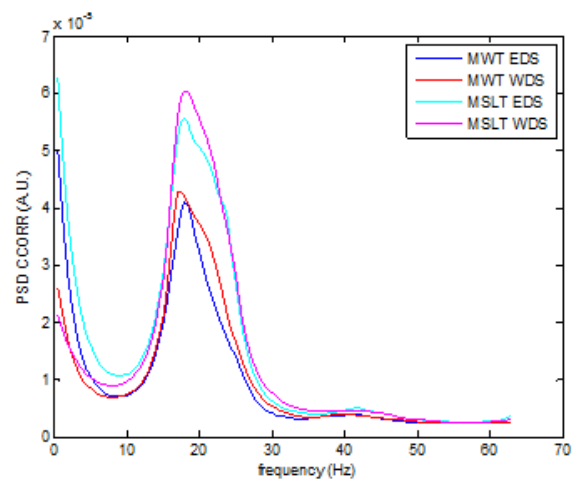

(b)

Fig. 2. Channel F3-O2, $\beta$ band. Averaging of function CCORR $(\tau)$ and (b) its power spectrum $\operatorname{CSD}(f)$ with respect to all MWT and MSLT of all EDS and WDS patients. 


\subsection{Analysis of the first 60-second window at the beginning of MSLT and MWT}

The best results in study 1 were found for $\operatorname{CSDmF}(\beta)$ of ACORR in O2 channel, yielding Sen $\geq 60$, Spe $>75$ and $A U C>0.75$ during all the MSLT tests, as it can be seen in table 1 . As examples, figure 3 shows the evolution of the mean value of $\operatorname{CSDmF}(\beta)$ of ACORR in O2 channel with respect to MWT and MSLT. It can be noted that ACORR calculated on EEG in $\beta$ band of WDS patients presents higher power in higher frequencies than EDS patients (figure 3 ) for all the tests.

Furthermore, $m(\beta)$ of CCORR between F3-O2 channels(Table 2) gives $S e n=70 \%$ and $S p e=95 \%, A U C=$ 0.840 in MSLT3, and Sen $=80 \%$ and Spe $=85 \%, A U C=0.815$ in MSLT5. Figure 4 shows the evolution of the mean value of $m(\beta)$ of CCORR between F3-O2 channels with respect to MWT and MSLT. A strong nonlinear coupling of EEG in $\beta$ band between F3 and O2 is presented during MSLT in EDS patients as it can be observed in figure 4.

In this first study, the best traditional measures applied to the current database were the power spectral in $\theta$ band $\left(P_{\theta}\right)$ in F3 channel in study 1 during MWT5 yielding Sen=65\% and Spe=70\%, AUC $=0.675$.

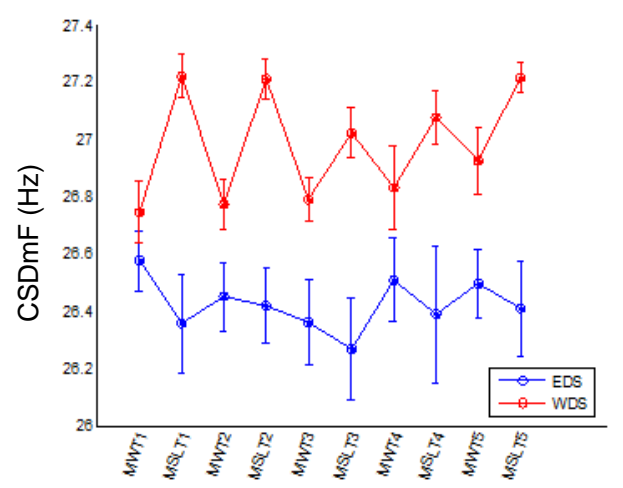

Fig. 3. Study 1: mean value and standard error of $\operatorname{CSDmF}(\beta)$ of $A C O R R$ in $\mathrm{O} 2$ channel.

Table 1. Study 1. Individual analysis of MSLT and MWT trials. Statistical analysis results between EDS and WDS group: $\operatorname{CSDmF}(\beta)$ of ACORR in channel O2

\begin{tabular}{|c|c|c|c|c|}
\hline Tests & p-value & $\operatorname{Sen}(\%)$ & Spe $(\%)$ & $A U C$ \\
\hline MWT1 & $0.1719^{\text {n.s. }}$ & 60 & 70 & 0.627 \\
\hline MSLT1 & 0.0001 & 65 & 85 & 0.857 \\
\hline MWT2 & 0.0468 & 60 & 80 & 0.685 \\
\hline MSLT2 & 0.0001 & 75 & 90 & 0.875 \\
\hline MWT3 & 0.0315 & 60 & 80 & 0.700 \\
\hline MSLT3 & 0.0006 & 70 & 80 & 0.820 \\
\hline MWT4 & 0.0223 & 50 & 75 & 0.712 \\
\hline MSLT4 & 0.0155 & 60 & 85 & 0.725 \\
\hline MWT5 & 0.0133 & 55 & 75 & 0.730 \\
\hline MSLT5 & $<0.0001$ & 60 & 100 & 0.890 \\
\hline
\end{tabular}

n.s., non-statistical significant

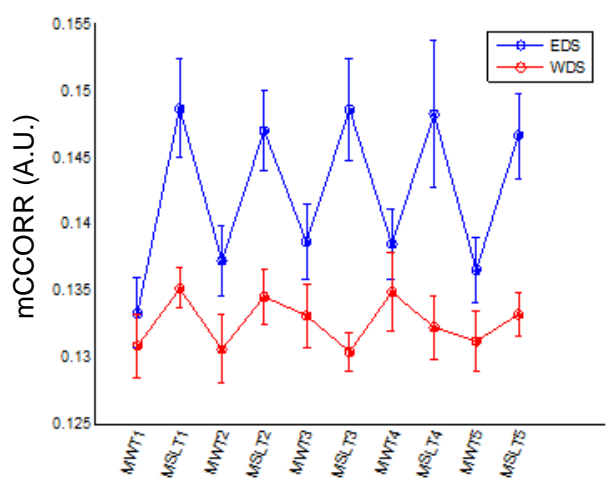

Fig. 4. Study 1: mean value and standard error of $m(\beta)$ of CCORR between F3-O2 channels. 
Table 2. Study 1. Individual analysis of MSLT and MWT trials. Statistical analysis results between EDS and WDS group:

\begin{tabular}{lllll}
\multicolumn{5}{c}{$m(\beta)$ of CCORR between F3-O2 channels } \\
\hline Tests & $p$-value & Sen $(\%)$ & Spe $(\%)$ & $A U C$ \\
\hline MWT1 & $0.715^{\text {n.s. }}$ & 50 & 60 & 0.535 \\
MSLT1 & 0.0016 & 70 & 85 & 0.792 \\
MWT2 & $0.1333^{\text {n.s. }}$ & 70 & 55 & 0.640 \\
MSLT2 & 0.0033 & 70 & 70 & 0.772 \\
MWT3 & $0.1806^{\text {n.s. }}$ & 45 & 60 & 0.625 \\
MSLT3 & 0.0002 & 70 & 95 & 0.840 \\
MWT4 & $0.2616^{\text {n.s. }}$ & 50 & 60 & 0.605 \\
MSLT4 & 0.0077 & 60 & 70 & 0.747 \\
MWT5 & $0.2733^{\text {n.s. }}$ & 60 & 55 & 0.602 \\
MSLT5 & 0.0007 & 80 & 85 & 0.815 \\
\hline \multicolumn{5}{l}{ n.s., non-statistical significant }
\end{tabular}

Regarding study 2, table 3 shows the ACORR and CCORR measures that have given both Sen and Spe $\geq 75$ $\%$ and $A U C \geq 0.8$ and the best traditional measures applied to the current database. It can be noted that the discrimination performance of the correntropy measures of study 1 were improved, evidencing better results during MSLT in study 2. Occipital region of EEG (O1 and O2) gave the best discrimination performance, the mean frequency of the ACORR spectrum $(\operatorname{CSDmF}(\beta))$ gave the higher Sen, Spe and AUC. This measure evidences that lower frequencies in the $\beta$ band prevail more in EDS group. The trend of $m F(\beta)$ in O1 channels reflects the behavior of $\operatorname{CSDmF}(\beta)$ but with lower Sen, Spe, and AUC. The values of CCORR measures indicate that the nonlinear coupling in $\beta$ band between occipital (O1, O2) and frontal (F3, F4) regions is stronger in EDS patients than WDS in MSLT tests.

Table 3. Study 2. ACORR and CCORR measures that have given the best performances differentiating the EDS from the WDS groups during the first minute of the tests, with the patient still awake.

\begin{tabular}{|c|c|c|c|c|c|c|}
\hline Measures & $E D S(m \pm S E)$ & $W D S(m \pm S E)$ & p-value & $\operatorname{Sen}(\%)$ & Spe(\%) & $A U C$ \\
\hline \multicolumn{7}{|l|}{ ACORR } \\
\hline \multicolumn{7}{|l|}{ MSLT trials } \\
\hline \multicolumn{7}{|l|}{ Channel F4 } \\
\hline$F D(T B)$ & $0.5314 \pm 0.0416$ & $0.5972 \pm 0.0473$ & 0.0003 & 80 & 80 & 0.833 \\
\hline \multicolumn{7}{|l|}{ Channel O1 } \\
\hline$F D(\beta)$ & $0.7907 \pm 0.0152$ & $0.8109 \pm 0.0051$ & $<0.0001$ & 75 & 95 & 0.908 \\
\hline $\operatorname{CSDmF}(\beta)$ & $26.54 \pm 0.5218$ & $27.21 \pm 0.2136$ & $<0.0001$ & 75 & 90 & 0.898 \\
\hline \multicolumn{7}{|l|}{ Channel O2 } \\
\hline $\operatorname{CSDmF}(\beta)$ & $26.37 \pm 0.5495$ & $27.15 \pm 0.2358$ & $<0.0001$ & 80 & 100 & 0.943 \\
\hline$F D(\beta)$ & $0.7869 \pm 0.0155$ & $0.8098 \pm 0.0062$ & $<0.0001$ & 85 & 95 & 0.935 \\
\hline \multicolumn{7}{|l|}{ CCORR } \\
\hline \multicolumn{7}{|l|}{ MSLT trials } \\
\hline \multicolumn{7}{|l|}{ Channels F3-C4 } \\
\hline $\min (\beta)$ & $0.1308 \pm 0.0181$ & $0.1120 \pm 0.0104$ & 0.0002 & 75 & 90 & 0.840 \\
\hline \multicolumn{7}{|l|}{ Channels F3-O2 } \\
\hline$m(\beta)$ & $0.1478 \pm 0.0116$ & $0.1331 \pm 0.0062$ & $<0.0001$ & 80 & 90 & 0.885 \\
\hline $\max L(\beta)$ & $0.1624 \pm 0.0132$ & $0.1469 \pm 0.0060$ & $<0.0001$ & 75 & 95 & 0.865 \\
\hline \multicolumn{7}{|c|}{ TRADITIONAL MEASURES } \\
\hline \multicolumn{7}{|l|}{ MSLT trials } \\
\hline \multicolumn{7}{|l|}{ Channel 01} \\
\hline$m F(\beta)$ & $21.84 \pm 1.61$ & $23.30 \pm 1.66$ & 0.0083 & 70 & 65 & 0.745 \\
\hline \multicolumn{7}{|l|}{ Channels O2-F3 } \\
\hline Cf SEF50(TB ) & $20.93 \pm 4.66$ & $25.84 \pm 5.44$ & 0.0051 & 70 & 70 & 0.750 \\
\hline
\end{tabular}

Table 4 shows the ACORR and CCORR measures that have given the best performances (Sen and Spe $\geq 75$ $\%$ and $A U C \geq 0.85$ ) and the best traditional measures in study 3 . The multivariable study 3 does not help to improve significantly the Sen and Spe of ACORR compared to study 2, while the Spe of CCORR is increased. 
Table 4. Study 3. Multi-variable discriminant function between measures in MWT with their equivalent in MSLT;

\begin{tabular}{|c|c|c|c|}
\hline \multicolumn{4}{|c|}{ EDS vs. WDS Discrimination } \\
\hline Measures & $\operatorname{Sen}(\%)$ & Spe $(\%)$ & AUC \\
\hline \multicolumn{4}{|l|}{ ACORR } \\
\hline \multicolumn{4}{|l|}{ Channel F3 } \\
\hline$C S D m F(T B)$ & 75 & 85 & 0.883 \\
\hline \multicolumn{4}{|l|}{ Channel 01} \\
\hline $\operatorname{CSDmF}(\beta)$ & 75 & 85 & 0.893 \\
\hline \multicolumn{4}{|l|}{ Channel O2 } \\
\hline $\operatorname{CSDmF}(\beta)$ & 85 & 90 & 0.955 \\
\hline$F D(\beta)$ & 80 & 95 & 0.940 \\
\hline \multicolumn{4}{|l|}{ CCORR } \\
\hline \multicolumn{4}{|c|}{ Channels F3-F4 } \\
\hline $\operatorname{CSDmF}(\beta)$ & 75 & 90 & 0.863 \\
\hline \multicolumn{4}{|c|}{ Channels F3-O2 } \\
\hline$m(\beta)$ & 75 & 95 & 0.900 \\
\hline $\max L(\beta)$ & 75 & 100 & 0.885 \\
\hline \multicolumn{4}{|c|}{ Channels F4-01 } \\
\hline $\max L(\beta)$ & 75 & 95 & 0.870 \\
\hline \multicolumn{4}{|c|}{ Channels F4-O2 } \\
\hline $\min (\beta)$ & 80 & 80 & 0.883 \\
\hline$m(\beta)$ & 75 & 90 & 0.878 \\
\hline \multicolumn{4}{|c|}{ TRADITIONAL MEASURES } \\
\hline \multicolumn{4}{|l|}{ Channel 02} \\
\hline$m F(\beta)$ & 80 & 70 & 0.747 \\
\hline \multicolumn{4}{|c|}{ Channels F4-O2 } \\
\hline$C f m(\delta)$ & 75 & 55 & 0.750 \\
\hline
\end{tabular}

\subsection{Analysis of Sleep Onset Proximity}

In this last study, in order to analyze the behavior of the non-linear measures in the entire recording as sleep onset (SO) approaches, we analyzed measures calculated in each of the $60 \mathrm{~s}$ sliding windows during the entire tests (MWT and MSLT). In the proximity to sleep onset of the MSLT, a significantly high number of windows showed statistical differences between groups in both $\operatorname{CSDmF}(\beta)$ of ACORR in $\mathrm{O} 1$ and $\mathrm{O} 2$ channels and $\mathrm{m}(\beta)$ of CCORR between F3-O2 channels (figure 5 ). The differences observed were similar to those reported at the first $60 \mathrm{~s}$ window of MSLT naps, where EDS patients showed lower frequencies in $\beta$ band using ACORR than in WDS group, in both $\mathrm{O} 1$ and $\mathrm{O} 2$ channels. Furthermore, more coupling between frontal and occipital zone is observed in EDS patients during the last $200 \mathrm{~s}$ before the SO.

The time-windows in which the statistical analysis between EDS and WDS patients gave p-value $<0.05$ and $A U C>0.75$ are represented by black squares and black triangles, respectively. Statistical differences between the two groups occur for a significantly high number of windows in waking state during MSLT2 and MSLT4. It was also found that in all MSLT, Sen and Spe were above or equal to $65 \%$ and AUC were above 0.7 when the mean value in all the windows of $\operatorname{CSDmF}(\beta)$ in $\mathrm{O} 2$ channel was taken into account, yielding $\operatorname{Sen}=80 \%$, Spe $=80 \%$ and $A U C=0.85$ in MSLT5. The results of the traditional measures in this study indicated that EDS group presents higher coherence than WDS group in $\delta$ band between channels F3-F4 during MWT and that the power in $\theta$ band of EDS group is higher than WDS group. However they Sen, Spe were never above $75 \%$ and $A U C$ was never above 0.75 .

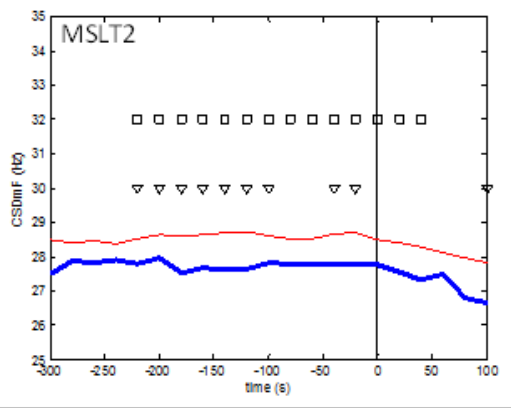

(a)

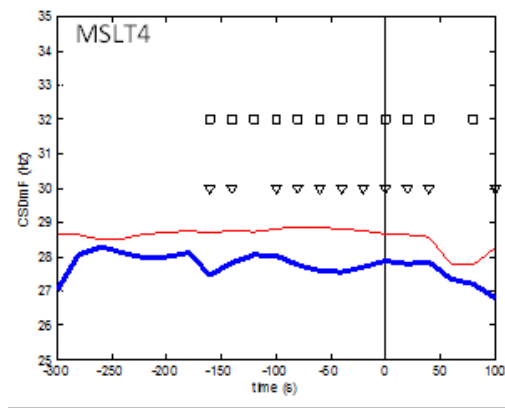

(b) 


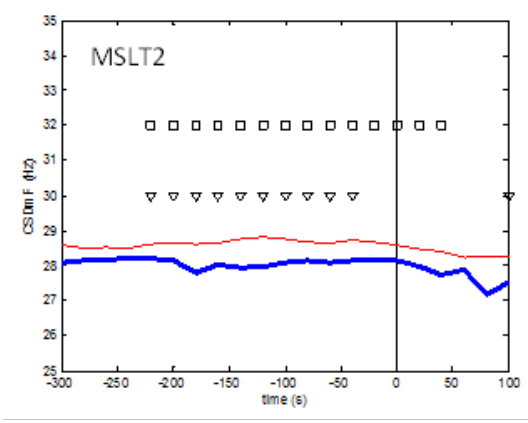

(c)

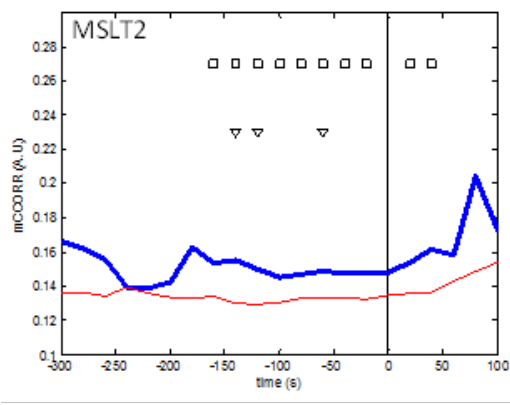

(e)
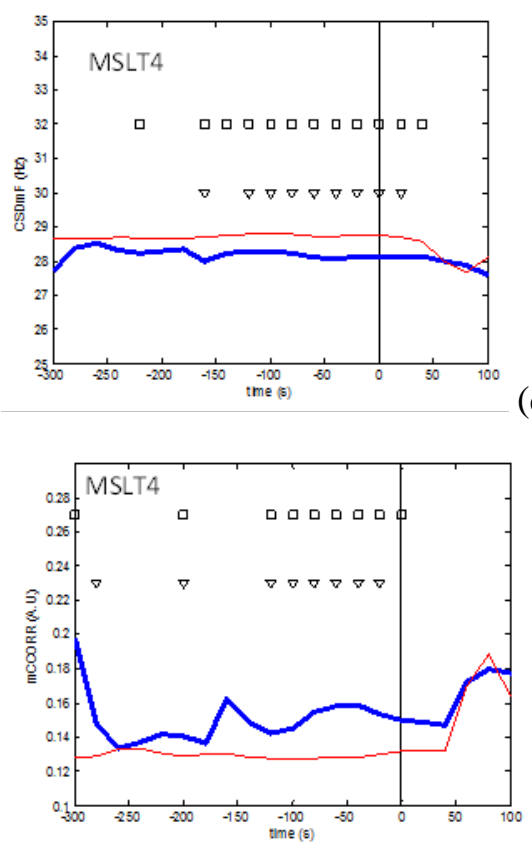

(f)

Fig. 5. Evolution of the mean value of (a, b) $\operatorname{CSDmF}(\beta)$ related to ACORR in $\mathrm{O} 1$ channel, (c, d) $\operatorname{CSDmF}(\beta)$ related to ACORR in $\mathrm{O} 2$ channel and (e, $\mathrm{f}) m(\beta)$ between F3-O2 channels related to CCORR of all the patients, EDS patients in blue and WDS patients in red. The measures were calculated by sliding $60 \mathrm{~s}$ windows with step of $20 \mathrm{~s}$ during MSLT2 and MSLT4 trials. The sleep onset is represented in $\mathrm{t}=0 \mathrm{~s}$ with the vertical black line. The results of statistical analysis between EDS vs. WDS patients for each window are shown by squares and triangles that indicate the time instants in which p-value $<0.05$ and $A U C>0.75$, respectively.

\section{Discussion}

Nonlinear dynamics analysis has been performed on EEG recorded in two groups of patients (EDS and WDS) who underwent two tests, MSLT and MWT. Several measures were calculated on ACORR and CCORR applied on $60 \mathrm{~s}$ EEG windows in waking states in order to characterize the two groups. The most remarkable results by both ACORR and CCORR in the discrimination of EDS and WDS patients were obtained in $\beta$ band during MSLT. Our results show that the best Sen, Spe and AUC was observed in ACORR measures applied on occipital region (O2) and in CCORR measures between interhemispheric region (O2-F3, O1-F4), central and occipital regions (O2-C4) and across the central line (O1-F3, O2-F4).

Previous studies showed that wakefulness before falling asleep is electrophysiologically characterized by the abrupt decrease in $\alpha$ and $\beta$ activity in EEG and the beginning of synchronized activity (De Gennaro et al 2005). The progressive synchronization of the electroencephalogram is expressed by a centro-frontal prominence within the $\delta / \theta$ frequency range starting about $60 \mathrm{~s}$ before sleep onset (SO) and lasted across the entire 5 min interval after SO (De Gennaro et al 2005). Other studies also showed that the $\beta$ band revealed a low level of EEG power that linearly decreased and was prevalent at the occipital scalp location (De Gennaro et al 2001). Similarly, figures 5a-d show that the tendency of the mean frequency ACORR spectrum in occipital zone calculated in $\beta$ band decreases more in EDS patients than in WDS when approaching the SO, evidencing statistical differences between EDS and WDS group in MSLT2 and MSLT4. This behavior is associated with an increasing of the coupling between frontal and occipital zone as it can be seen in figures 5e and $5 f$. Furthermore, the results of CCORR denote that this coupling is stronger for EDS patients than for WDS in $\beta$ band between the frontal (F3, F4) and central (C3, C4) zones and occipital zones (O1, O2) for MSLT (p-value<0.005). Furthermore, results shown in Table 2 demonstrated that the power spectrum of ACORR in $\beta$ band presents lower frequencies in EDS group than WDS in waking windows for MSLT in the occipital zones (p-value $<0.005$ ).

Similarly to the present work, Picot and co-workers (2009) developed an algorithm based on a mean comparison test applied on the EEG power calculated in $\alpha$ band for detecting drowsiness in drivers yielding 
more than $80 \%$ of accurate detection. However, their work assumed that the subject starts driving in an alert state and the mean comparisons are based on the EEG signal obtained at the beginning of the recording. In this way, if the person does not start driving in an alert state, the algorithm does not consider the changes. Garcés Correa et al. (2014) yield similar results to those obtained in Picot et al. (2009), using a multi-modal analysis of the EEG to detect the drowsiness stage which is independent of the initial state of the driver. Their results have been obtained processing EEG records from poly-somnographic studies in contrast to the present work, where the combination between MWT and MSLT takes into account two different situations in which the patient try to be alert or spontaneously fall asleep.

In another research, Bonnet and Arand (2005) compared the heart rate in two healthy young adult groups with (MSLT $<7 \mathrm{~min}$ ) and without daytime sleepiness (MSLT $>10 \mathrm{~min}$ ). Using some frequency-domain measures they show differences between alert and drowsy group. However, in the current work, we took into account EEG during wakefulness in people with and without sleepiness and focused in the awake period before sleep onset, defined as the first three consecutive epochs of stage I sleep.

Other studies have analyzed the EEG during the process of entering sleep, but taking sleep onset as the time of the first sleep spindle (De Gennaro et al. 2001, De Gennaro et al. 2005 ), which is much later than what we have considered in the present study. In a previous work (Melia et al. 2014), statistical differences between EDS and WDS group were found in the frontal zone during MWT trials using time-frequency representation of EEG. However, the discrimination performances were $A U C<0.9$ and Sen and Spe never above 90\%, lower than the results yielded with correntropy measures.

The selection of 60-second window at the beginning of each MWT and MSLT trials might be a limitation of the current study since longer EEG segment gets more fluctuation and might contain any kind of EEG noise or randomness. However, this permits to fix the same window length for all the patients obtaining a good compromise between the sample size and the minimum latency in which the patients were awake in all the trials. We hypothesized that patients with shorter sleep latencies in each of the two tests, therefore those with more sleepiness would have changes in their EEG already detectable in the initial parts of the recordings, even with the patient apparently awake and that more fragments with the patient sleepy would be present as compared with those without sleepiness. These windows were selected at the beginning of each MWT and MSLT in order to ensure to have all the patients in the same condition. Analyzing all the results it is possible to infer that correntropy function robustness against some kind of noise and its capability to characterize the nonlinear properties of the EEG signals permit to outperform the traditional measures perturbed by the random fluctuation of the EEG. As an example, in figure 4, it can be seen that the behavior of the $m(\beta)$ of CCORR in EDS patients has a regular tendency during the whole day. It shows almost the same mean value in all the MSLT trials, always higher than the mean value of all the MWT trials. Similar behavior is also observed in figure 3 for $\operatorname{CSDmF}(\beta)$ of ACORR of WDS patients. Since it is really unlikely to associate these tendencies with randomness or noise of the recorded signals, we can assume that these measures are able to reflect the physiological changes between the different situation of the MWT and MSLT trials. In the same way, these measures are able to characterize the different sleepiness levels of the EDS and WDS groups.

Furthermore, study 4 was performed in order to have a better validation of the findings obtained in the 60second windows at the beginning of each MWT and MSLT. The statistical differences observed in the 60second window between EDS and WDS groups were observed in a quite large number of windows during MSLT2 and MSLT4 (figure 5). Since these results are in concordance with the findings of study 1, it is possible to assume that the statistical differences observed in the window at the beginning of these trials are preserved in the subsequent windows.

The mean frequency of ACORR in $\beta$ band for WDS group in MSLT presents changes respect to the same measure in MWT (Figure 3). These changes are also observed in the CCORR in $\beta$ band for EDS group in MSLT respect to MWT (Figure 4). The different results between MWT and MSLT trials might be due to the fact that in MSLT trial both groups closed the eyes while WDS group patients were widely in eyes opened condition in MWT trials. In MWT trials, statistical differences were not observed between EDS and WDS groups. It can be assumed that the changes in the complexity and nonlinear coupling in the EEG and in ocular movements related to the task of staying awake do not reflect different sleepiness states between the two groups. On the other hand, the influence of the ocular movement on the results in MSLT test is assumed to be not significant because all patients were in eyes closed condition. Furthermore, the statistical differences in ACORR measures were observed mostly on occipital region where the alteration in the EEG recording due to eye movements is minimized. The circadian oscillations that are present when a test is performed at different times during the day might be a further reason of the differences in the results between each MSLT and each 
MWT. The two groups showed different sleep latency reflecting different levels of sleepiness in the different period of the day in which the trial was performed. In general, both groups of patients presented higher sleep latency as well as less sleepiness in the first trial (MSLT1 and MWT1) due to the previous sleepy night. In the third and fifth trials, both groups of patients exhibited higher sleepiness levels due to the after lunch time somnolence and the tiredness at the end of the day, respectively. These factors might influence the changes of the EEG measures and the statistical performances.

Regarding the classical EEG measures, similarly to ACORR and CCORR measures, the highest performances was observed in study 2 and study 3 for the mean frequency in $\beta$ band of channel O1 and O2. Comparing the results of the traditional temporal and frequency linear measures with the ACORR and CCORR results in tables 1-4, it can be noted that in each study the best ACORR and CCORR measures have higher values of Sen, Spe, and AUC than the best traditional measures. This demonstrates the improvements of the proposed method due to the capability of correntropy functions of characterizing non-linear EEG features that traditional measures cannot detect. However, these results need a retrospective study to be designed including data recorded on a higher number of patients. This future work will permit to develop correntropy based index for the automatic detection of the excessive sleepiness.

\section{Conclusions}

A new method to detect sleepiness was introduced by studying the non-linear dynamics of the EEG signal by correntropy based measures. In general, patients with excessive sleepiness presented less complexity in $\beta$ band than patients without sleepiness in all EEG channels during MSLT associated with an ACORR power spectrum higher in lower frequencies in the occipital zones. Non-linear coupling in $\beta$ band between the frontal (F3, F4) and central (C3, C4) zones and occipital zones (O1, O2) are stronger in patients with excessive sleepiness than patients without sleepiness. Measures of ACORR power spectrum and CCORR yield the classification performances Sen $>80 \%$, Spe $>80 \%$ and $A U C>0.85$. The classification performances of CCORR have increased by combining measures from MWT with their equivalents from MSLT (study 3). Similar statistical differences were observed in the measures applied to a significant number of windows during the entire recording.

In conclusion, ACORR and CCORR measures applied to a 60-second window of EEG improved the characterization of patients suffering excessive daytime sleepiness, yielding discrimination performances higher than traditional measures.

\section{Acknowledgment}

This work was supported within the framework of the CICYT grant TEC2010-20886, the FIS PI07/0318 to MS (co-financed by FEDER) and the Research Fellowship Grant FPU AP2009-0858 from the Spanish Government. CIBER of Bioengineering, Biomaterials and Nanomedicine is an initiative of ISCIII.

\section{References}

Acharya U R, Faust O, Kannathal N, Chua T and Laxminarayan S 2005 Non-linear analysis of EEG signals at various sleep stages Computer Methods and Programs in Biomedicine 80 37-45

Alonso J F, Mananas M A, Romero S, Hoyer D, Riba J and Barbanoj M J 2010 Drug effect on EEG connectivity assessed by linear and nonlinear couplings Human brain mapping 31 487-497

Andrews-Hanna J R, S.Reidler J, Huang C and Buckner R L 2010 Evidence for the default networks role in spontaneous cognition Journal of Neurophysiology 104 322-335

Andrzejak R G, Lehnertz K, Moormann F, Rieke C, David P and Elger C E 2001 Indications of nonlinear deterministic and finite-dimensional structures in time series of brain electrical activity: dependence on recording region and brain state Phys. Rev. 64061907

Arand D, Bonnet M, Hurwitz T, Mitler M, Rosa R and Sangal R B 2005 The clinical use of the MSLT and MWT Sleep 28 123-44

Bonnet M H and Arand D L 2005 Performance and cardiovascular measures in normal adults with extreme MSLT scores and subjective sleepiness levels Sleep 28 685-693

Carskadon M A, Dement W C, Mitler M M, Roth T, Westbrook P R, and Keenan S 1986 Guidelines for the Multiple Sleep Latency Test (MSLT): a standard measure of sleepiness Sleep 9 519-524

Chiou J C, Ko L W, Lin C T, Hong C T, Jung T P, Liang S F and Jeng J L 2006 Using novel MEMS EEG sensors in detecting drowsiness application, Proc. of Biomedical Circuits and Systems Conference. London pp 33-36 
Chouvarda I, Rosso V, Mendez M O, Bianchi A M, Parrino L, Grassi A, Terzano M and Cerutti S 2011 Assessment of the EEG complexity during activations from sleep Computer methods and programs in biomedicine 104 e16-e28

De Gennaro L, Ferrara M and Bertini M 2001 The boundary between wakefulness and sleep: quantitative electroencephalographic changes during the sleep onset period Neuroscience 107 1-11

De Gennaro L, Vecchio F, Ferrara M, Curcio G, Rossini P M and Babiloni C 2005 Antero-posterior functional coupling at sleep onset: Changes as a function of increased sleep pressure Brain Research Bulletin 65 133-140

Fell J, Röschke J and Beckmann P 1993 Deterministic chaos and the first positive Lyapunov exponent: a nonlinear analysis of the human electroencephalogram during sleep Biological cybernetics 69 139-146

Fu J, Li M and Lu B L 2008 Detecting drowsiness in driving simulation based on EEG, Autonomous SystemsSelf-Organization, Management, and Control 1 21-28

Garcés Correa A, Orosco L and Laciar E 2014 Automatic detection of drowsiness in EEG records based on multimodal analysis Medical Engineering \& Physics 2 244-249

Gunduz A and Principe J C 2009 Correntropy as a novel measure for nonlinearity tests Signal Processing $\mathbf{8 9}$ 14-23

Gusnard D A, Akbudak E, Shulman G L and Raichle M E 2001 Medial prefrontal cortex and self-referential mental activity: Relation to a default mode of brain function Proceedings of the National Academy of Sciences of the United States of America 98 4259-4264

Kingshott R N, Engleman H M, Deary I J and Douglas N J 1998 Does arousal frequency predict daytime function? Eur Respir J 12 1264-70

Jeong J 2004 EEG dynamics in patients with Alzheimer’s disease Clin. Neurophysiol. 115 1490-1505

Johnson R R, Popovic P D, Olmstead R E, Stikic M, Levendowski D J and Berka C 2011 Drowsiness/alertness algorithm development and validation using synchronized EEG and cognitive performance to individualize a generalized model Biological Psychology 87 241-250

Jung T and Makeig S 1994 Estimating levels of alertness from EEG Proc. IEEE- EMBS Conf pp 103-1104

Lin C T, Wu R C, Jung T P, Liang S F and Huang T Y 2005 Estimating driving performance based on EEG spectrum analysis EURASIP Journal of Applied Signal Processing 19 3165-3174

Lin C T, Pal N R, Chuang C Y, Jung T P, Ko L W and Liang S F 2008 EEG-based subject- and sessionindependant drowsiness detection: an unsupervised approach Neural Networks IJCNN (IEEE World Congress on Computational Intelligence) pp 3448-3454

Littner M R, Kushida C and Wise M 2005 Practice parameters for clinical use of the multiple sleep latency test and the maintenance of wakefulness test Sleep 28 113-21

Lombardi C, Parati G, Cortelli P, Provini F, Vetrugno R, Plazzi G and Vignatelli L 2008 Daytime sleepiness and neural cardiac modulation in sleeprelated breathing disorders Journal of sleep research 17 263270

Makeig S and Inlow M 1993 Lapses in alertness: coherence of fluctuations in performance and EEG spectrum Electroencephalography and Clinical Neurophysiology 86 23-35

McCullagh P and Nelder J A 1990 Generalized Linear Models. (New York: Chapman \& Hall).

Melia U, Guaita M, Vallverdú M, Clariá F, Montserrat J M, Vilaseca I, Salamero M, Gaig C, Caminal P, Santamaria J 2014 Characterization of Daytime Sleepiness by Time-Frequency Measures of EEG Signals Journal of Medical and Biological Engineering , doi:10.5405/jmbe.1823

Ohayon M M 2008 From wakefulness to excessive sleepiness: what we know and still need to know Sleep Med Rev 12 129-141.

Pradhan N and Sadasivan P K 1996 The nature of dominant Lyapunov exponent and attractor dimension curves of EEG in sleep, Computers in biology and medicine 26 419-428

Picot A, Charbonnier S and Caplier A 2009 Monitoring drowsiness on-line using a singleencephalographic channel In: de Mello CAB, editor. Biomedical Engineering. Croatia: In Tech pp 145-64

Richardson G S, Carskadon M A, Flagg W, Van den Hoed J, Dement W C and Mitler M M 1978 Excessive daytime sleepiness in man: multiple sleep latency measurement in narcoleptic and control subjects Electroencephalogr Clin Neurophysiol. 45 621-627

Sabeti M, Katebi S and Boostani R 2009 Entropy and complexity measures for EEG signal classification of schizophrenic and control participants Artificial intelligence in medicine 47 263-274

Sangal RB, Thomas L and Mitler MM 1992 Maintenance of wakefulness test and multiple sleep latency test. Measurement of different abilities in patients with sleep disorders Chest 101 898-902

Santamaria I, Pokharel P P and Principe J C 2006 Generalized correlation function: definition,properties and application to blind equalization IEEE Transactions on Signal Processing 54 2187-2197 
Santamaria J and Chiappa K H 1987 The EEG of drowsiness in normal adults Journal of clinical Neurophysiology 4 327-382

Smith M E, McEvoy L K and Gevins A 2002 The impact of moderate sleep loss on neurophysiologic signals during working-memory task performance Sleep 25 784-794

Stam C J 2005 Nonlinear dynamical analysis of EEG and MEG: review of an emerging field Clin.Neurophysiol. 116 2266-2301

Subasi A and Erçelebi E 2005 Classification of EEG signals using neural network and logistic regression Comput Methods Programs Biomed 78 87-99

Swarnkar V, Abeyratne U and Hukins C 2010 Objective measure of sleepiness and sleep latency via bispectrum analysis of EEG Medical \& Biological Engineering \& Computing 48 1203-1213

Thorpy M J, Westbrook P, Ferber R, Fredrickson P, Mahowald M, Perez-Guerra F, Reite M, and Smith P 1992 The clinical use of the Multiple Sleep Latency Test Sleep 15 268-276

Vanhaudenhuyse A, Demertzi A, Schabus M, Noirhomme Q, Bredart S, Boly M and Laureys S 2010 Two distinct neuronal networks mediate the awareness of environment and of self Journal of Cognitive Neuroscience 1 1-9

Vuckovic V, Radivojevic N, Chen A C and Popovic D 2002 Automatic recognition of alertness and drowsiness from EEG by an artificial neural network Medical Engineering \& Physics 24 349-360

Wilson B J and Bracewell T D 2000 Alertness monitor using neural networks for EEG analysis In: Proc. Neur. Net Signal Process X (ISPS) 2 814-820

Xu J W, Bakardjian H, Cichocki A and Principe J C 2007 A New Nonlinear Similarity Measure for Multichannel Biological Signals Proceedings of International Joint Conference on Neural Networks, Orlando, Florida, USA 1 12-17

Yeo M V, Li X, Shen K and Wilder-Smith E P 2009 Can SVM be used for automatic EEG detection of drowsiness during car driving? Safety Science 47 115-124 